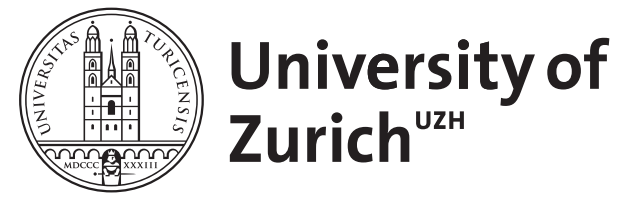

\title{
Recording primate vocalizations
}

Geissmann, T ; Parsons, S

DOI: https://doi.org/10.1017/CBO9780511921643.018

Posted at the Zurich Open Repository and Archive, University of Zurich

ZORA URL: https://doi.org/10.5167/uzh-58582

Book Section

Published Version

Originally published at:

Geissmann, T; Parsons, S (2011). Recording primate vocalizations. In: Setchell, J M; Curtis, D J. Field and laboratory methods in primatology: A practical guide, second edition. Cambridge: Cambridge University Press, 287-303.

DOI: https://doi.org/10.1017/CBO9780511921643.018 
Geissmann, T., and Parsons, S. (2011). Recording primate vocalizations. In Setchell, J. M., and Curtis, D. J. (eds.) Field and laboratory methods in primatology: A practical guide, second edition. Cambridge University Press, Cambridge, pp. 287-303.

THOMAS GEISSMANN AND STUART PARSONS

16

Recording primate vocalizations

I N T R O D U C T I O N

Ornithologists have been exploring the possibilities and the methodology of recording and archiving animal sounds for many decades. Primatologists, however, have only relatively recently become aware that recordings of primate sound may be just as valuable as traditional scientific specimens such as skins or skeletons, and should be preserved for posterity (Fig. 16.1). Audio recordings should be fully documented, archived and curated to ensure proper care and accessibility. As natural populations disappear, sound archives will become increasingly important (Bradbury et al., 1999).

Studying animal vocal communication is also relevant from the perspective of behavioural ecology. Vocal communication plays a central role in animal societies. Calls are believed to provide various types and amounts of information. These may include, among other things: (1) information about the sender's identity (e.g. species, sex, age class, group membership or individual identity); (2) information about the sender's status and mood (e.g. dominance, fear or aggressive motivation, fitness); and (3) information about relevant events or discoveries in the sender's environment (e.g. predators, food location). When studying acoustic communication, sound recordings are usually required to analyse the spectral and temporal structure of vocalizations or to perform playback experiments (Chapter 11).

This chapter describes how to record nonhuman primate vocalizations. We begin with definitions of some technical terms, then provide information on the advantages and disadvantages of various types of equipment (sound recorders, microphones and other equipment). Next we discuss methods for recording ultrasound, before ending with tips for better recordings of primate vocalizations, relevant to both the 


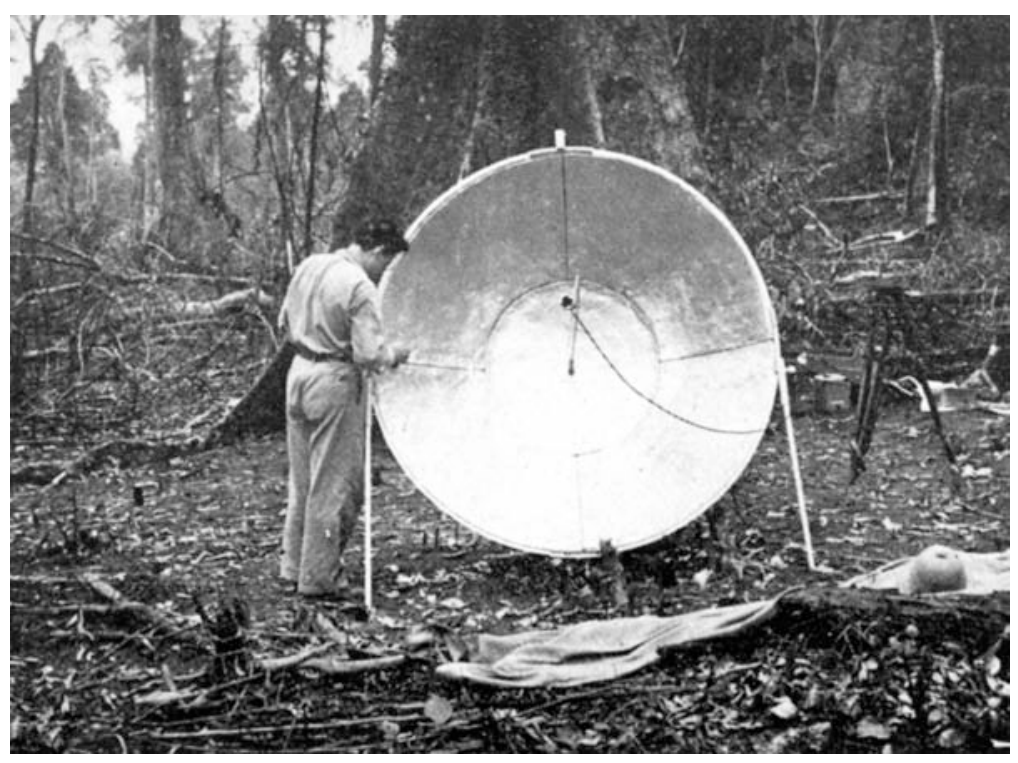

Fig. 16.1 Clarence R. Carpenter and his parabolic reflector microphone in Chiang Dao, Chiang Mai, Thailand, 1937. This installation was used to record the calls of wild gibbons on disk. Photo from Carpenter (1940, p. 26; Johns Hopkins Press, Baltimore)

field and captivity. Ornithologists and chiropterologists have to deal with very similar problems, and we recommend their introductory texts for further study (e.g. Budney \& Grotke, 1997; Kroodsma et al., 1996; Parsons \& Szewczak, 2009; see also Useful Internet sites).

We do not provide information on the prices for sound equipment. As a rule, these prices change markedly over the years, and retailers' prices differ widely. We recommend that you compare prices on the Internet before buying. The specific models of sound equipment that are produced also change rapidly. Some of the recorders and microphones recommended in the first edition of this book (Geissmann, 2003) went out of production even before the book was published. The same will apply to the products discussed in the present chapter. Digital devices tend to be particularly short-lived and products are frequently replaced by follow-up models or completely discontinued. We recommend that you check the product palette on the Internet before deciding on a particular model. We do not describe methods and equipment required for sound analysis, but you can find information on that topic elsewhere (Charif et al., 1995, 2004; Hopp et al., 1998; Pavan, 2006b; Parsons \& Szewczak, 2009). 
T E C H N I C A L T E R M S

Sound is a wave phenomenon. Sound waves are oscillations in atmospheric pressure.

The amplitude of a sound wave is proportional to the change in pressure during one oscillation. The greater the amplitude of the wave, the more energy it transmits. In sound waves, the amplitude relates to volume, so the greater the amplitude, the higher the energy and the louder the sound.

Frequency $f$ in wave motion is the number of waves that pass through a given point per second. It is the reciprocal of the time $T$ taken to complete one cycle (the period), or 1/T. Frequency is usually expressed in units called hertz $(\mathrm{Hz})$. One hertz is equal to one cycle per second; one kilohertz $(\mathrm{kHz})$ is $1000 \mathrm{~Hz}$.

Ultrasound is sound with a frequency greater than the upper limit of human hearing. Human hearing is normally limited to frequencies between about 12 and $20000 \mathrm{~Hz}$.

\section{SOUND RECORDERS}

An ideal recorder records a signal without alterations, by matching its dynamic and frequency range and by preserving all its features. Traditional analogue tape-recorders are not perfect. They degrade the signals they record by adding hiss, distortion, frequency response alterations, speed variations, print-through effects and drop-outs. Digital recorders do not have these problems. Within the dynamic range and the frequency limits (owing to the number of bits and sampling frequency they use), they record and reproduce signals with great accuracy, low noise, flat frequency response and no speed variations. Nevertheless, the quality of the signal recorded by good analogue tape-recorders is sufficient for most purposes in bioacoustic studies.

The majority of primate species live in tropical rainforests. Any lengthy stay in this kind of environment puts considerable stress on electronic equipment (see also Chapter 17). If making sound recordings is a crucial component of your study, choose sturdy and humidiyresistant recording machines over highly sophisticated but delicate equipment. Also consider acquiring two complete sets of soundrecording equipment, because the equivalent replacement of a broken machine may not available anywhere in the country where you are working, and a repair may require replacement parts that may be equally unavailable. Similarly, some digital audio recorders are capable 
of saving to two types of media simultaneously (e.g. hard disk and compact flash) thus providing an immediate backup of all recordings.

We discuss several sound recording formats that are no longer supported by the industry (e.g. analogue, DAT) or that are not an option for bioacoustic analyses because they use sound compression (e.g. MP3 recorders) below. They are included here because the reader may have access to some of this equipment and may want to know whether it may still be useful (and some of it is). If you plan to buy new equipment anyway then proceed directly to the section on 'Hard-disk and solidstate recorders'.

\section{Analogue tape-recorders (audio)}

The analogue tape-recorders that were most suited for field studies are not produced any more, but as they include some of the most durable field equipment ever produced for sound recording, we still recommend them, if you can find them. We mention tape-recorders only briefly, but you can find more detailed comments on these machines in Geissmann (2003).

Avoid the use of noise reduction features such as Dolby or DBX during recording, as they add distortion and limit the high frequencies of many sounds. If possible, use a tape-recorder with adjustable sensitivity for signal intensity. The automatic level control (ALC) that is found on many cheaper portable cassette recorders is often ineffective for recording sounds in the field. Unfortunately, there always were very few portable machines with adjustable sensitivity. The use of ALC may also render recordings unusable for playback studies or for analysis of source levels.

The best machines included the small Sony Walkman WM-D6, the TCM-5000EV, and the larger Sony TC-D5M and TC-D5 Pro II. The Walkman's advantage is its small size, whereas the larger machines come equipped with speakers. Several portable cassette recorders with adjustable sensitivity were also produced by Marantz (e.g. PMD-201, PMD-222 and CP-430) and resembled their Sony counterparts in most respects.

The decision between stereo and mono depends on what your recording will be used for. For most field recordings, stereo is an unnecessary complication. However, if you are interested in recording vocal interactions between several individuals or groups, then a stereo machine is preferable. A second audio channel can also be useful for recording researcher comments via a lapel microphone or similar. 
Making effective written notes while recording can often be difficult, and audio comments can be invaluable in these situations. Be sure you transcribe all audio comments soon after making recordings.

\section{Digital Audio Tape (DAT)}

This is another technology that has virtually disappeared. The DAT recording system stores the sound information in a binary code, thereby making it immune to speed errors, tape noise (hiss) and non-linear frequency response problems. The DAT recorder delivers a sound quality slightly better than compact disc (CD; $48 \mathrm{kHz}$ versus $44 \mathrm{kHz}$, often with a greater dynamic range) in a small, easy to use and easy to store, long-duration, tape-based format. Recordings made to DAT can be transferred directly to computer, without the need to redigitize. This minimizes any potential degradation of the signal. Both the machines and the recording tapes are very difficult to obtain now. Unfortunately, DATs are generally very sensitive to high humidity and, therefore, not reliable in some recording environments such as rainforest. Most DAT recorders have a 'dew' or humidity sensor built in that shuts the machine down whenever high humidity is detected. Most DAT machines will operate for only two hours per battery charge, and only accept rechargeable cadmium batteries, a problem when you are working in areas with no or unreliable access to line power. Because of these limitations, we do not recommend the use of DAT recorders.

MP3, Minidisc (MD), etc.

Avoid working with technologies that remove information from the sound. Of these, MP3 (Moving Picture Experts Group Layer 3) is currently the most popular format, but the same reservations apply to formats like MiniDisc, Digital Compact Cassette, MP4 (Moving Picture Experts Group Layer 4), or Liquid Audio and VQF (also called TwinVQ, transform-domain weighted interleave vector quantization). All these technologies use sound compression algorithms that discard sound details that appear to be non-audible to humans, but may be of biological relevance to primates. For this reason, no compressed or filtered sounds should be used for playback experiments. Input filtering and data compression strategies distort the signal in subtle ways that make it more difficult to analyse and render accurate frequencyrelated measurements impossible. It may also lead to perceptible distortion if you make multiple copies. Any such technology will 
change (i.e. degrade) the sound you are recording and make your recordings unsuitable for sound analysis.

\section{Hard-disk and solid-state recorders}

Direct-to-hard-disk recording by using a laptop computer would be an alternative with great advantages, but normal portable computers are not usually designed to survive long in a tropical climate or under the rough treatment that is unavoidable when following primates through the rainforest. We do not, therefore, discuss this method further here, but see Pavan (2006a) for more information on the topic.

A better option for field use is offered by small, compact recorders that record on internal hard disks or on memory cards such as the compact flash memories also used in digital cameras (Chapter 17). A pivotal criterion for choosing one of these digital recorders is whether they offer the option to store sound not only in compressed format such as MP3 (which is not recommended, see above), but also in uncompressed formats such as WAV (Waveform Audio File Format) or AIFF (Audio Interchange File Format). Macs and most PCs can read these files and edit them (with appropriate software). These recorders range from pocket-sized to book-sized. Some models record up to 4 channels simultaneously and some can sample at rates of up to $192 \mathrm{kHz}$. Some are not designed for scientific recording, but are more music-oriented.

The most promising technology for carrying out sound recordings in the field are solid-state recorders that record sound on a flashcard and thus use no moving mechanical parts. Examples are Flash Recorders of the PMD series from Marantz (e.g. PMD 620, 660, PMD 670, PMD 671), but it appears that they are no longer produced. Recorders made by Sound Devices, such as the 722 and 744, are capable of recording to compact flash, hard disk, or both. Other, very small, digital recorders that use internal flash memory include the Sony PCM series (PCM-D1, PCM-M10/B, PCM-D50) or the ZOOM H4n. These come equipped with electret condenser microphones. The built-in microphones may not be sufficient for field recordings of primates, unless the primates are calling close to the recorder, but the recorders have input jacks for external directional microphones.

Other recorders suited for field recordings include following: Edirol R1, R-4/R-4Pro and R-09HR; M-Audio MicroTrack II; Fostex FR-2 and FR-2LE; Tascam HD-P2. Each device has its own features, advantages and disadvantages that you will need to evaluate according to 
research needs and budget. Moreover, new and interesting devices are likely to appear in the near future.

Specifications given by manufacturers may be confusing, especially those related to the noise of the microphone pre-amplifiers. Most are suitable for recording loud concerts, but the self-noise of the microphone and of the recorder may add an annoying hiss that limits the possibility of capturing low-level sounds in quiet environments. Among the recorders mentioned above, the Sound Devices $7 \mathrm{xx}$ series 'has the best reputation for reliability, flexibility and overall sound quality, in particular for the low noise mic preamplifiers' (Pavan, 2006a).

\section{MICROPHONES AND PARABOLIC REFLECTORS}

If possible, use a directional microphone or a microphone with a parabolic reflector (also called a parabola). Directional microphones improve sound collection in nature considerably by reducing unwanted ambient noise and sounds from other directions, and are the most useful in bioacoustic field recordings. Various names are in use for microphones with various degrees of directionality. Here, we differentiate between shotgun or ultradirectional microphones and semi-directional microphones. Parabolic reflectors improve sound collection by giving emphasis to the sounds coming frontally.

\section{Shotgun or ultradirectional microphones}

A shotgun microphone is a cardioid microphone fitted with an interference tube on its frontal face. It is characterized by a flat frequency response and is less sensitive to wind and handling noise, but offers a lower sensitivity, than a microphone mounted in a parabola. The interference tube cancels off-axis signals, while the on-axis signals reach the microphone's diaphragm without attenuation or gain. As a rule, shotgun microphones work in mono only, so you would need two of them if you wanted to make a stereo recording.

There are many brands of high-quality microphone available, but they are relatively expensive. As a simple rule, the degree of directionality in shotgun microphones correlates with their length (and, usually, with their price). Unfortunately, longer microphones are not very handy in dense tropical forests - they get in the way and are more easily damaged than shorter microphones. We have tested various models of the Sennheiser line of microphones. They are expensive 
but they have proven to be very durable, exhibit low noise figures, and are relatively immune to high humidity.

Sennheiser's combination of the ME 66 microphone capsule and the K6 pre-amp/power unit using an AA battery is particularly useful in the field. This system has a total length of $32 \mathrm{~cm}$. For better directionality, you can plug the longer ME 67 into the K6, for a combined length of $44 \mathrm{~cm}$. The top-of-the-range Sennheiser shotgun microphones (e.g. MKH 416-P48U3 [25 cm], MKH 418-S [28 mm], MKH 60 [28 mm], MKH 70-1 [41 cm]) have particularly flat frequency responses but tend to be more expensive. Note that you also need special microphone cables with appropriate connectors for the selected recorder for some Sennheiser directional microphones (available separately).

Directional microphones are also produced by a number of other companies including Audio-Technica (e.g. AT 8015, AT 8035, AT 875R, AT 897), Røde (NTG-1, NTG-2, NTG-3) and Edirol (CS-50). Some are less expensive than the Sennheisers but still exhibit good directionality.

On windy days, you may need to add a rubber foam windscreen to your directional microphone. This functions to keep the wind turbulence as far away from the microphone surface as possible. Some microphones come equipped with a windscreen, in others it is an extra. In some cases, it is relatively expensive (about US\$30), although it is basically just a tight-fitting glove of rubber foam for your microphone. A similar windscreen of your own manufacture should work reasonably well and costs almost nothing. Be careful that your windscreen does not selectively filter (remove) certain frequencies from your recordings.

\section{Semi-directional microphones}

Semi-directional microphones produced for video cameras (Chapter 17) are typically less directional than the shotgun microphones, but are cheaper and smaller. They may suffice if you are recording 'loud-calls' of primates or if you are studying captive animals, where background noise is frequently less of a problem and where the microphone can usually be positioned closer to the vocalizing animals. However, the degree of directionality and other quality characteristics vary strongly (Wölfel \& Schoppmann, 1994). One of us (TG) has used semi-directional microphones to record loud vocalizations of various primates, including gibbons (Hylobatidae), leaf monkeys (Presbytini), macaques (Macaca) and indris (Indri indri), in the field with good results. Usually, these videomicrophones can be plugged directly into the sound recorders with no 
special connecting cable needed (unlike some of the directional microphones from Sennheiser), but we recommend buying an extension cable (1.5 m) for field use. Example semi-directional microphones are Hama's RMZ series (RMZ-10, RMZ-12, and RMZ-14), Røde VideoMic Directional Video Condenser Microphone, the video microphone from Sennheiser (MKE 400), the DM series from Canon (DM-50, DM-100), and AudioTechnica's ATR 6550. Be careful to select a microphone with a frequency range suitable for your needs. The frequency range of some of the cheaper video microphones does not exceed $10 \mathrm{kHz}$, which makes them less suited to tape-recording primates with high-pitched vocalizations (e.g. callitrichids).

If you are working with analogue recorders, the microphone should never be too close to the cassette recorder while recording; otherwise you might have the noise of the machine on your soundrecordings.

\section{Parabolic reflectors}

A parabolic reflector focuses incoming sound waves that are parallel to its axis onto a single point, the focus, where a microphone is placed. Whereas a shotgun microphone simply screens off sound from directions other than the sound source, a parabolic reflector can actually amplify the sound from the target direction. It also acts as a highpass filter, especially for low-frequency environmental sounds. The effectiveness is determined by the diameter of the reflector in relation to the wavelength of the sound. Gain and directivity increase proportionally as the diameter: wavelength ratio increases. For wavelengths larger than the diameter of the parabola, the response is predominantly that of the microphone itself. As the wavelengths become smaller than the parabola diameter, gain and directivity increase with the frequency. Common diameters are $45 \mathrm{~cm}, 60 \mathrm{~cm}$ and $90 \mathrm{~cm}$, with directionality starting at about 750, 550 and $375 \mathrm{~Hz}$, respectively. We only recommend the larger dishes for primate studies: they are able to cover the frequency range of calls produced by the smaller primate species, at least. Many larger species, however, produce calls that go well below $375 \mathrm{~Hz}$ which would require a parabola diameter above $90 \mathrm{~cm}$. Obviously, this would difficult to use in tropical forests, even if some plastic parabola (Telinga) can be rolled up for travelling. Parabola may be of better use in stationary recording sites or in more open habitat.

A variety of parabolic reflectors are available, made of metal, fibreglass or clear plastic. Some manufacturers, such as Telinga and Saul 
Mineroff Electronics, sell complete systems that include a parabolic reflector with a microphone and shock-mount system. If you are working on a tight budget, you might consider building a parabolic reflector yourself. You can add some directionality to any non-directional microphone with a large bowl- or umbrella-shaped dish. Position the microphone in the focal point of the reflector, which, fortunately, is not very narrowly defined. The sound is concentrated in this area, and increases sensitivity.

\section{OTHER EQUIPMENT}

Many ornithologists recommend the use of good-quality headphones that allow you to listen while recording, making it easier to aim the microphone, as well as giving a clear idea of the quality of the recording being made. We find it inconvenient, however, to monitor the vocal activities of primates, often calling from several directions at the same time, with earphones on.

If you are making analogue recordings, we recommend using quality audio-cassettes (e.g. TDK, Maxell). Much cheaper cassettes are available, but they may yield fuzzy recordings and some frequency levels may be under-represented. Of the available cassettes (types I, II and IV), type II tape offers the best high-frequency response and the lowest signal-to-noise ratio. Interesting vocalizations tend to occur exactly when the cassette is full, and you will miss them while swapping cassettes. We therefore prefer cassettes that allow you to record for 90 or 100 minutes (instead of 60 minutes). Shorter tapes, on the other hand, are more time accurate, because the tape is thicker.

Always carry a set of new, non-rechargeable batteries with you, for emergencies. Animals often decide to vocalize after you have spent hours recording background noise and all your rechargeable batteries have expired. In such cases, an emergency set of batteries will come in handy.

\section{U L T R A S O U N D}

A number of small primates, e.g. pygmy marmosets (Cebuella), galagos (Galago), mouse lemurs (Microcebus) and lorises (Nycticebus), have been shown to emit ultrasound vocalizations; others, e.g. the greater bamboo lemur (Prolemur), have been reported to produce sounds with harmonics that can extend into the ultrasound range (Bergey \& Patel, 2008; Cherry et al., 1987; Glatston, 1979; Pariente, 1974; Pola and Snowdon, 
1975; Zietemann, 2000; Zimmermann, 1981). Fundamental frequencies of some note types of the grey mouse lemur (Microcebus murinus) reach maximum values of about $45 \mathrm{kHz}$ (Zietemann, 2000). Because detecting, recording and analysing ultrasound vocalizations requires special equipment, such calls may be more widespread among small primates (and other small mammals) than has been documented so far. Fortunately, much research is being carried out on ultrasound calls of bats, and scientists wishing to study ultrasound vocalizations of primates can greatly benefit from exploring the expertise of, and the technologies being used by, bat researchers (see Parsons \& Szewczak, 2009 for a recent review).

\section{Bat detectors}

Bat detectors or ultrasound detectors were developed to provide researchers with instruments to study bat echolocation, but are also used for research on other small mammals and grasshoppers. Bat detectors are based on both analogue and digital techniques to detect and record ultrasounds and transform them into sounds audible to the human ear. The detectors available on the market use three main systems: heterodyne frequency shifting, frequency division and time expansion.

Heterodyne detectors and frequency division (or count-down) detectors are real time methods (i.e. you hear the sound from the detector at the same time as it is emitted by the bat). Heterodyne detectors remove all spectral and temporal information from the sounds they transform and are thus of limited use. However, they are small and light and so useful for determining whether an animal is producing ultrasound or not. Use caution in interpreting the output of such detectors, as they often have a wide listening bandwidth (up to $16 \mathrm{kHz}$ ). This means that vocalizations may appear to be ultrasonic when they are not. Heterodyne detectors are also only capable of monitoring a narrow bandwidth at one time, so may miss the vocalizations of animals calling outside the monitored frequencies.

Frequency-division detectors lower the frequency of a vocalization by reducing the number of cycles that it contains (usually by a factor of 10). At the same time, many also remove amplitude information. This inevitably reduces the amount of information contained in the vocalization, making such detectors of limited use experimentally. However, the detectors' ability to work in real-time, their retention of the spectral content of the vocalization, albeit at a reduced resolution, 
and their ability to monitor a wide range of frequencies simultaneously makes them an excellent monitoring tool.

If you want to study the frequency content of ultrasonic vocalizations in detail, you will need a time-expansion detector (e.g. Pettersson D240x, D980, D1000X; Ultra Sound Advice: U30 + PUSP). Time expansion relies on the inverse relation between time and frequency: if time is doubled, frequency is halved. Time expansion detectors function by sampling a sound at a high rate, then playing it back at a lower rate, thus effectively stretching it in time. The result is a sound lowered in frequency, but that retains all the temporal, spectral, and intensity information of the original sound. The method is similar to making a high-speed tape recording of the sound and then playing it back at a lower speed. Clearly, this is not a real-time conversion method, but it does offer a number of benefits over and above heterodyne and frequency-division methods. Since the signal is stretched out in time, it is possible to hear details of the sound not audible with other types of detector (for example, you can actually hear frequency differences in single short pulses).

\section{Digital data acquisition}

It has recently become possible to record ultrasound in real time, without the need for transformation. This is ideal if you wish to make high-quality recordings suitable for detailed analysis in the lab, and for playback in the field. Ultrasound is acquired by a suitable microphone, digitized at a very high rate, and then either held in RAM or saved to hard-disk or some other storage medium (e.g. compact flash). The simplest and cheapest option for acquiring ultrasound directly is a digital bat detector. These possess microphones with relatively flat frequency responses that extend beyond $150 \mathrm{kHz}$. As mentioned previously, many are capable of transforming the ultrasound, but several also give the researcher access to the untransformed ultrasound (e.g. Pettersson D980, D1000x; UltraSound Advice U30). Detectors such as the D1000x will also save the ultrasound to compact flash, meaning that you do not need immediate access to a laptop computer. However, all others require the ultrasound output to be sent to a specialist recorder capable of sampling at rates sufficiently high to capture the ultrasound.

Some solid-state recorders (e.g. those made by Sound Devices) are capable of sampling at rates up to $192 \mathrm{kHz}$ and so can acquire most vocalizations and their harmonics. Newer laptop computers are also equipped with built-in sound cards capable of sampling at similar rates. 
If this is not the case, then you will need separate USB or PCMCIA sound acquisition cards (e.g. the DAQCard 6062E made by National Instruments). If you use a laptop computer to record the ultrasound, you will need software to control the process. Software programs that can interface with built-in sound cards as well as USB and PCMCIA-based systems include Raven (Cornell University Laboratory of Ornithology), BatSound Pro (Pettersson Elektronik AB) and AviSoft Recorder (Avisoft Bioacoustics).

Specialist solutions are also available that allow you to acquire multiple channels directly to laptop computer in real-time, and are field-portable (e.g. those made by AviSoft Bioacoustics). These come with microphones, amplifiers, digital acquisition systems and the software to run them.

Acquisition of ultrasound in real time has two primary disadvantages: cost and storage. Ultrasound recording systems are significantly more expensive than those suited to lower frequencies and, owing to the high sampling rates used, file sizes are often large. If your budget allows for the purchase of such a system, ensure that you also purchase sufficient storage to hold, and back up, all your recordings.

\section{RECORDING PROCEDURE}

Get into the habit of making frequent comments while recording your animals. This will be very helpful later when you analyse your recordings. For instance, if several individuals are vocalizing together, it is often impossible to determine who made which vocalization later on. Record information about the identity, sex and age of a vocalizing animal, and the context in which each vocalization is produced. It is usually impossible to describe a long vocalization bout in detail from memory after the recording has been made. Therefore, do it as the vocal bout goes on but try to speak during intervals between vocalizations. Of course, it is best not to voice input at the same time as your target animal is calling.

Always narrate 'stop' or some other indication that the recorder is turned off before stopping. The lack of critical voiced information on tape, especially where one recording ends and another begins, is one of the greatest failings of the beginner. If you interrupt a tape-recording ('cut'), say so on the tape. Otherwise, it is often difficult to detect that the recorder was paused, and confusion can occur during later analysis. If you continue recording the same or new target subjects following the cut, add some narration to say so. 
Make long, uninterrupted recordings. Vocalizations tend to occur when you think they won't. If you are waiting for a vocalization to occur, it may be worth while recording continuously, even while your animals are not vocalizing. By doing so, you will catch complete vocalizations or vocal bouts, of which you would otherwise miss the beginning.

Hold the microphone very firmly and steadily. Even moving your fingers may interfere with recordings. If possible, use a tripod to hold the microphones. Small field-portable models are cheap, light and durable. Alternatively, if the calling animal is stationary and known to produce long calling bouts, orient the microphone in the correct direction on a branch (this usually requires an extension cable). This avoids the risk of disturbing the sound recordings when your fingers and arms get tired during a long vocal bout. Moreover, it frees your hands and allows you to take notes, consult your watch, or take compass bearings during the recording.

Record as close to the subject as possible. Directional microphones and parabolic reflectors are no substitute for proximity. Very soft, quiet vocalizations are lost in ambient noise if you are too far away from the animal. Occasionally, though, closer may not be better. If you encounter a loud sound source, such as calling cicadas, somewhere between you and your target animals, going closer would bring you closer to the cicadas as well. In this case, it may be better to change position.

Two special recommendations if you are carrying out analogue audio-recordings:

1. If no vocalizations occur, rewind your cassette from time to time. If you already have vocalizations on the same side of the cassette on which you intend to record, set the rotation-counter to zero before starting to record again. This will allow you to rewind to the end of your last recording when you have been taperecording for some time with no vocalizations.

2. When the side of an audio-cassette is filled with recordings, always break out the record-enabling chip on the upper left, thin side of the cassette, to ensure that you do not accidentally record on this side again and delete important material. You may decide to give provisional field numbers to your tapes and give them final inventory numbers when you are back in the laboratory. In any case, it is important that tapes can be identified at any time and referred to in your field notes. 
Be consistent in your methods and the way that you record data. That way, someone else listening to your recordings later can learn your pattern, in your absence, and retrieve important information.

Recordists frequently overlook the importance of adequate documentation of recordings, often obtained with painstaking effort and sometimes at great expense. Lack of documentation seriously weakens the scientific value of your sound recordings. After making the recording, narrate the following information on tape:

1. Recordist and equipment configuration: name of the recordist, type of recorder, microphone and parabolic reflector.

2. Identification of target subject and the degree of certainty: name of species, type of contact (heard only, seen), number of target subjects on record, sex and age class of target animal(s) or group composition (if known), approximate distance of subject from microphone, cross-references if same individual can be heard on previous recordings.

3. Time and location: date; time at the beginning and the end of recording, time at important occasions (swapping cassettes, calls of new individuals, etc.), location (accurate locality, district, province, country).

4. Other information: type of habitat, weather conditions, behaviour of subject, identification of other sounds in the background.

Make a written edit of your tape as soon as possible after recordings are completed. Keep a journal of your recordings detailing their contents. You can also add information that was not narrated onto the sound recording. Editing recordings takes a lot of time but makes your collection much more usable. It is a good idea to listen to your recordings while the day's work is still fresh in your memory, and make a written record of what you have recorded.

Keep your personal collection of wildlife sounds well documented and in one place. Label both your sound media (e.g. files, cassettes, DVDs) and your storage boxes clearly.

Review recordings as frequently as possible, preferably at the end of each day. Often, recordings that appear perfect in the field are less so when reviewed later.

Finally, digitize recordings to computer as soon as possible. This provides a necessary backup against degradation of analogue recording media and an additional copy of digital media. We use the 3-2-1 protocol for backing up our recordings: three backups, in two separate 
places, one of which is off-site. Few things are more tragic than the loss of a life-time's work due to theft of a computer or loss of media.

\section{ACKNOW LE D G E M E N T S}

We are grateful to Deborah J. Curtis, Joanna M. Setchell, Sabine Schmidt, Robert Dallmann and Marina Davila Ross for reading and commenting on earlier versions of this manuscript.

\section{R E F E R E N C E S}

Bergey, C. \& Patel, E.R. (2008). A preliminary vocal repertoire of the greater bamboo lemur (Prolemur simus): classification and contexts. Nexus 1, 69-84.

Bradbury, J., Budney, G. F., Stemple, D. W. \& Kroodsma, D. E. (1999). Organizing and archiving private collections of tape recordings. Anim. Behav. 57, 1343-4.

Budney, G.F. \& Grotke, R.W. (1997). Techniques for audio recording vocalizations of tropical birds. Ornithol. Monogr. 48, 147-63.

Carpenter, C. R. (1940). A field study in Siam of the behavior and social relations of the gibbon (Hylobates lar). Comp. Psychol. Monogr. 16, 1-212.

Charif, R. A., Mitchell, S. \& Clark, C.W. (1995). Canary 1.2 User's Manual. Ithaca, NY: Cornell Laboratory of Ornithology.

Charif, R. A., Clark, C. W. \& Fristrup, K. M. (2004). Raven 1.2.1 User's Manual. Ithaca, NY: Cornell Laboratory of Ornithology.

Cherry, J. A., Izard, M. K. \& Simons, E. (1987). Description of ultrasonic vocalizations of the mouse lemur (Microcebus murinus) and the fat-tailed dwarf lemur (Cheirogaleus medius). Am. J. Primatol. 13, 181-5.

Geissmann, T. (2003). Tape-recording primate vocalisations. In Field and Laboratory Methods in Primatology: A Practical Guide, ed. J.M. Setchell \& D. J. Curtis, pp. 228-38. Cambridge: Cambridge University Press.

Glatston, A. R. (1979). Reproduction and behaviour of the lesser mouse lemur (Microcebus murinus, Miller 1777) in captivity. Ph.D. thesis, University College, University of London.

Hopp, S. L., Owren, M. J. \& Evans, C. S. (eds.) (1998). Animal Acoustic Communication Sound Analysis and Research Methods. Berlin: Springer Verlag.

Kroodsma, D. E., Budney, G. F., Grotke, R. W. et al. (1996). Natural sound archives: guidance for recordists and a request for cooperation. In: Ecology and Evolution of Acoustic Communication in Birds, ed. D. E. Kroodsma \& E. H. Miller, pp. 474-86. Ithaca, NY: Cornell University Press.

Pariente, G.F. (1974). Importance respective du reperage visuel et auditif (absence d'écholocation) chez Microcebus murinus. Mammalia 38, 1-6.

Parsons, S. \& Szewczak, J. M. (2009). Detecting, recording, and analyzing the vocalizations of bats. In Ecological and Behavioral Methods for the Study of Bats, ed. T. H. Kunz \& S. Parsons, pp. 91-111. Baltimore, MD: Johns Hopkins University Press.

Pavan, G. (2006a). Instruments and Techniques for Bioacoustics. Università degli Studi di Pavia. Centro Interdisciplinare di Bioacustica e Ricerche Ambientali (CIBRA). http://www.unipv.it/cibra/edu_equipment_uk.html\#reco, accessed 28 Oct. 2009. 
Pavan, G. (2006b). Software for Sound Analysis. Università degli Studi di Pavia. Centro Interdisciplinare di Bioacustica e Ricerche Ambientali (CIBRA). http://www-3.unipv.it/cibra/res_software_uk.html, accessed 28 Oct. 2009.

Pola, Y.V. \& Snowdon, C.T. (1975). The vocalizations of pygmy marmosets (Cebuella pygmaea). Anim. Behav. 23, 825-42.

Wölfel, M. \& Schoppmann, J. (1994). Vergleichstest: Richtmikrophone - Lasst Mikros sprechen. VIDEOaktiv 1994, 4-7.

Zietemann, V. (2000). Artdiversität bei Mausmakis: Die Bedeutung der Akustischen Kommunikation. $\mathrm{PhD}$ thesis, Institut für Zoologie, Tierärztliche Hochschule Hannover.

Zimmermann, E. (1981). First record of ultrasound in two prosimian species. Naturwissenschaften 68, 531-2.

\section{US E F UL I N TERNET S ITES}

Internet sites with review texts on recording and analysing nature sounds: http://blb.biosci.ohio-state.edu/

http://zeeman.ehc.edu/envs/Hopp/sound.html

http://www.birds.cornell.edu/brp

http://www-3.unipv.it/cibra/ (see 'Bioacoustic Equipment') 
